\title{
Study on the Fluorescence Depolarization of a Chromophore Attached to Polymer Chain End II. Aggregation and Helix-Coil Transition Behavior of Poly $(\gamma$-benzyl L-glutamate) in Various Mixed Solvents
}

\author{
Hideharu UsHIKI and Itaru MITA* \\ Laboratory of Chemistry, Faculty of General Education, \\ Tokyo University of Agriculture and Technology, \\ 3-5-8, Saiwai-cho, Fuchu-shi, Tokyo 183, Japan \\ *Institute of Interdisciplinary Research, Faculty of Engineering, \\ The University of Tokyo, 4-6-1, Komaba, Meguro-ku, Tokyo 153, Japan
}

(Received March 21, 1984)

\begin{abstract}
The emission anisotropy of the fluorescence depolarization of rhodamine B chemically bound to the terminal amino group of poly( $\gamma$-benzyl L-glutamate) (PBLG) was measured in various helicogenic solvents (benzene, dioxane, etc.). The apparent mean rotational relaxation time of the chromophore attached to chain end corresponded to the aggregation behavior of PBLG in various helicogenic solvents. This shows that the terminal rhodamine $\mathrm{B}$ group as a chromophore is tightly bound to the PBLG chain end. The aggregation and helix-coil transition behavior of PBLG has been studied in various solvent mixtures (benzene-dichloroacetic acid, benzene- $N, N$-dimethylformamide, etc.). In the series of highly aggregation-solvents such as benzene and dioxane, PBLG is aggregated up to an $N, N$-dimethylformamide content of about 80 $90 \%$. The aggregation-breaking (dissociation) and helix-breaking ratios as parameters of aggregation and helix-coil transition behavior of PBLG are briefly discussed for various solvent mixtures.
\end{abstract}

\begin{abstract}
KEY WORDS Poly( $\gamma$-benzyl L-glutamate) / Rhodamine B Group / Fluorescence Depolarization / Emission Anisotropy / Aggregation / Dielectric Constant / Mean Rotational Relaxation Time / Helix-Coil Transition Behavior / Aggregation-Breaking (Dissociation) Ratio / HelixBreaking Ratio /
\end{abstract}

In our previous work, ${ }^{1}$ the fluorescence depolarization of the rhodamine $B$ group attached to the chain end of poly $(\gamma$-benzyl Lglutamate) (PBLG) was measured in chloroform (helicogenic solvent)-dichloroacetic acid (helix-breaking solvent) mixtures. When the composition of dichloroacetic acid is increased, the apparent helix-breaking ratio

$$
\begin{array}{r}
\left(b_{\mathrm{x}}=\left(a_{\mathrm{x}}-a_{\mathrm{CF}}\right) /\left(a_{\mathrm{DCA}}-a_{\mathrm{CF}}\right) \times 100(\%),\right. \\
\text { see eq } 6 \text { and } 7)
\end{array}
$$

in the vicinity of the terminal group of PBLG, increases gradually before leaping to $100 \%$ at a $60-75 \%$ dichloroacetic acid (DCA) content, indicating a gradual increase in the freedom of motion of the terminal group. In addition, the somewhat sharp increase in the helix-breaking ratio at $0-10 \%$ DCA may be ascribed to the dissociation of the head-to-tail type aggregation of PBLG often observed in helicogenic solvent. The technique based upon the fluorescence depolarization of chromophore attached to helix-polymer chain end is specially to the study of aggregation and helix-coil transition behavior in very dilute and semi-concentrated solutions, and liquid crystals. Thus far the behavior of chain end group of polypeptides has been studied only by the spin labeling technique (ESR). ${ }^{2,3}$ 
The fluorescence depolarization method, first cased by Perrin, ${ }^{4}$ has been reported by many authors in the study of biological science. This technique is very useful for analyzing the rotational Brownian motion of a fluorescent molecule or group in a certain environment. In the study of polymers, this technique has recently been used to investigate the dynamic behavior of polymer segments of a polystyrene gel, ${ }^{5}$ polyacrylamide, ${ }^{6}$ and anthryl group labeled to a polymer. ${ }^{7,8}$

Reviews on the conformation-dependent properties of synthetic polypeptides in the helix-coil transition region have been reported by Teramoto and Fujita. ${ }^{9,10}$ The helix-coil transition behavior of polypeptide has already been measured by the well-known methods (ORD,${ }^{11}$ viscosity, ${ }^{12}$ light scattering, ${ }^{13}$ dielectric relaxation, ${ }^{14} \mathrm{CD},{ }^{15,16}$ etc.). Moreover, the aggregation behavior of PBLG has been measured by well-known methods (dielectric relaxation, ${ }^{17-20} \mathrm{GPC}^{21}$ etc.) as well. Thus far, in the field of the aggregation and helix-coil transition behavior, the technique based on the labeled probe method has been reported by a few authors. ${ }^{1,22}$

In this work, aggregation and helix-coil transition behavior in the vicinity of rhodamine B chemically bound to the terminal amino group of PBLG is investigated in various helicogenic solvents and in the series of benzene, dioxane, chloroform-DCA or $N, N$ dimethylformamide (DMF) mixtures by the fluorescence depolarization probe method developed recently. ${ }^{1}$ In various helicogenic solvents, the apparent mean rotational relaxation time of the rhodamine B group attached to PBLG chain end varies in proportion to the dielectric constant of the solvents. Consequently, it is possible to estimate the aggregation behavior of PBLG by the fluorescence depolarization probe method. Finally, the aggregation behavior in the helix-coil transition region of PBLG is discussed briefly.

\section{EXPERIMENTAL}

Rhodamine B was attached to the chain end of prepared PBLG in advance from Lglutamate- $N$-carboxyanhydride as previously reported. ${ }^{1}$ The molecular weight of PBLG having a rhodamine $B$ group at the chain end (PBLG-RhB) was calculated from its intrinsic viscosity in DMF at $25^{\circ} \mathrm{C}$, and the content of rhodamine B group at the chain end of PBLG was determined from its fluorescence intensity. Preparations of the PBLG-RhB used are PBLG260RhB $\left(M_{w}=5.6 \times 10^{4}\right.$, content of chromophore; 20\%) and PBLG480RhB $\left(M_{w}=1.1 \times 10^{5}\right.$, content of chromophore; $22 \%){ }^{1}$ In the notation for the samples, the figures between PBLG and $\mathrm{RhB}$ indicate the approximate degree of polymerization (DP).

The fluorescence depolarizations of a polymer at a concentration ranging from $10^{-6} \mathrm{M}$ to $10^{-5} \mathrm{M}\left(0.01-0.1 \mathrm{~g} \mathrm{~d}^{-1}\right)$ were measured with a JASCO FP-550 spectrofluorometer with polarizing filters. Dotite-Luminasol-grade dioxane, benzene, chloroform, and DMF were used as solvents for the fluorescence depolarization measurements.

\section{RESULTS AND DISCUSSION}

\section{Measurements of Emission Anisotropy}

The emission anisotropy $r$ can be calculated from eq 1 ,

$$
\begin{aligned}
r & =\left(I_{\mathrm{vv}} I_{\mathrm{hh}}-I_{\mathrm{vh}} I_{\mathrm{hv}}\right) /\left(I_{\mathrm{vv}} I_{\mathrm{hh}}+2 I_{\mathrm{vh}} I_{\mathrm{hv}}\right) \\
& =\left(I_{\mathrm{vv}} G I_{\mathrm{vh}}\right) /\left(I_{\mathrm{vv}}+2 G I_{\mathrm{vh}}\right)
\end{aligned}
$$

where $I$ is the intensity of emitted light with $\mathrm{v}$ and $h$ as a suffix denoting the vertically and horizontally polarized lights. The first suffix corresponds to the incident light, and the second, to the emitted light. The $G\left(=I_{\mathrm{hv}} / I_{\mathrm{hh}}\right)$ factor in eq 1 is necessary to correct for the depolarization characteristics of the apparatus. The emission anisotropy $r$ is related to the mean rotational relaxation time $\rho$ of a 
chromophore by eq 2 ,

$$
r_{0} / r=1+(3 \tau / \rho)
$$

where $\tau$ is the excited singlet lifetime of the chromophore and $r_{0}$, the emission anisotropy in a rigid solution.

The ratio $\rho_{\mathrm{s}} / \rho_{\mathrm{p}}$ in each mixed solvent can be expressed by eq 3 ,

$$
\rho_{\mathrm{s}} / \rho_{\mathrm{p}}=\left\{\tau_{\mathrm{s}}\left(r_{0 \mathrm{p}} / r_{\mathrm{p}}-1\right)\right\} /\left\{\tau_{\mathrm{p}}\left(r_{0 \mathrm{~s}} / r_{\mathrm{s}}-1\right)\right\}
$$

Equation 3 can be rewritten as follows by assuming $r_{0 \mathrm{p}} \cong r_{0 \mathrm{~s}}$ and $\tau_{\mathrm{p}} \cong \tau_{\mathrm{s}}$,

$$
\rho_{\mathrm{s}} / \rho_{\mathrm{p}}=\left\{r_{\mathrm{s}}\left(r_{0 \mathrm{~s}}-r_{\mathrm{p}}\right)\right\} /\left\{r_{\mathrm{p}}\left(r_{0 \mathrm{~s}}-r_{\mathrm{s}}\right)\right\}
$$

where the suffixs $\mathrm{p}$ and $\mathrm{s}$ denote the polymer (PBLG-RhB) and small molecules (RhBNCS), respectively.

As already mentioned in the previous paper, ${ }^{1}$ changes of $r_{0}$ and $\tau$ of RhB-NCS (5isothiocyanatorhodamine $\mathrm{B}$ ) and PBLG-RhB with the composition of the mixed solvent are practically the same in various mixed solvents.

The fluorescence depolarization of PBLG$\mathrm{RhB}$ was measured at a polymer concentration of $0.1-0.5 \mathrm{~g} \mathrm{dl}^{-1}$. In the previous paper, ${ }^{1}$ we confirmed that the fluorescence quenching of the $\mathrm{RhB}$ group bound to PBLG by the polymer itself (amide group) is not important in this concentration range.

\section{Determination of $r_{0}$ of $R h B-N C S$}

To obtain the emission anisotropy $r_{0 \mathrm{~s}}$ of RhB-NCS in a rigid solution, the emission anisotropy $r_{\mathrm{s}}$ was measured in glycerine-water mixtures. The graph for $1 / r v s . T / \eta$ is shown in Figure 1. Here, the emission anisotropy $r$ is related to the rotational volume $V$ of chromophore and the viscosity of solvents $\eta$ by eq 5 ,

$$
r_{0} / r=1+(3 \tau / \rho)=1+(k \tau T / v \eta)
$$

where $k$ and $T$ are the Boltzman constant and an absolute temperature, respectively. Consequently, the intercept and slope in Figure 1 represent $r_{0}^{-1}$ and $k \tau / v r_{0}$, respectively. The values calculated by the least-squares method are obtained as follow: RhB-NCS $\left(r_{0}^{-1}=\right.$ $3.0, k \tau / v=1.8 \times 10^{-4} \rho$ (poise) $\mathrm{K}^{-1}$ ).

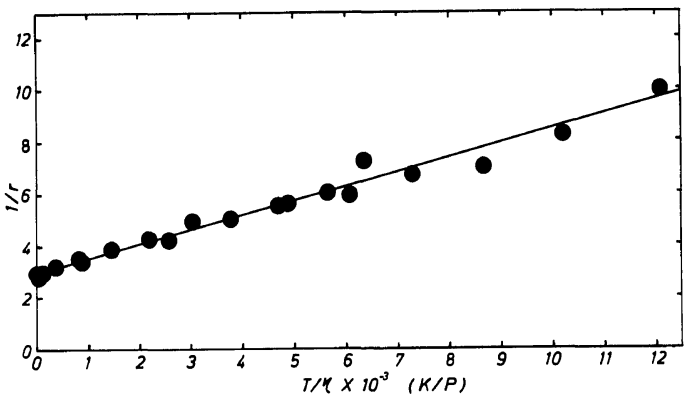

Figure 1. Graph for the reciprocal of the emission

\begin{tabular}{|c|c|c|c|c|c|c|}
\hline \multirow{2}{*}{ Solvent } & \multirow{2}{*}{$\varepsilon$} & \multirow{2}{*}{$\frac{\mathrm{RhB}-\mathrm{NCS}}{r^{-1}}$} & \multicolumn{2}{|c|}{ PBLG260RhB } & \multicolumn{2}{|c|}{ PBLG480RhB } \\
\hline & & & $r^{-1}$ & $\rho_{\mathrm{s}} / \rho_{\mathrm{p}}$ & $r^{-1}$ & $\rho_{\mathrm{s}} / \rho_{\mathrm{p}}$ \\
\hline Dioxane & 2.21 & 21 & 6.7 & 0.21 & 5.7 & 0.15 \\
\hline Benzene & 2.28 & 44 & 5.2 & 0.055 & 3.3 & 0.0073 \\
\hline Chloroform & 4.9 & 50 & 20 & 0.35 & 8.8 & 0.12 \\
\hline THF & 7.6 & 23 & 15 & 0.53 & & \\
\hline$m$-Cresol & 11.8 & 3.9 & 3.6 & 0.71 & & \\
\hline Pyridine & 12.4 & 20 & 17 & 0.79 & 10 & 0.41 \\
\hline Benzyl alcohol & 13.0 & 9.2 & 7.4 & 0.72 & 5.4 & 0.40 \\
\hline 2-Chloroethanol & 25.8 & 12 & 9.3 & 0.69 & & \\
\hline DMF & 36.8 & 18 & 14 & 0.74 & 8.0 & 0.33 \\
\hline DCA & & 7.2 & 6.9 & 0.94 & 2.5 & 0.85 \\
\hline
\end{tabular}
anisotropy of RhB-NCS, against glycerine-water mixtures.

Table I. Fluorescent depolarization of RhB-NCS and PBLG-RhB in various helix-solvents 
Measurement of Fluorescence Depolarization of $P B L G-R h B$ in Various Solvents

The $r^{-1}$ values for the emission anisotropy of PBLG-RhB and $\rho_{\mathrm{s}} / \rho_{\mathrm{p}}$ values calculated by eq 4 in various solvents at room temperature are shown in Table I along with the values of dielectric constant $\varepsilon$ of the solvents. Moreover, the dependence of $\rho_{\mathrm{s}} / \rho_{\mathrm{p}}$ on the dielectric constant of each solvent is shown in Figure 2. According to Sakamoto and Watanabe, ${ }^{24}$ PBLG is highly aggregated in dioxane (DX), benzene (BZ), and benzyl alcohol (BA), moderately aggregated in chloroform $(\mathrm{CF})$ and tetrahydrofuran (THF), slightly aggregated in $m$-cresol, molecularly dispersed in pyridine, 2chloroethanol, and $N, N$-dimethylformamide (DMF), and is randomly coiled in dichloroacetic acid (DCA).

When the $\rho_{\mathrm{s}} / \rho_{\mathrm{p}}$ value equals unity in Table I, the mean rotational relaxation time of a rhodamine B group attached to PBLG chain end manifests the same mobility as that of RhB-NCS, a small molecule. Since the $\rho_{\mathrm{s}} / \rho_{\mathrm{p}}$ in eq 4 cancels out the effect of the difference of the excited singlet lifetime of rhodamine B group and of the solvent viscosity in each solvent, a comparison of the values $\rho_{\mathrm{s}} / \rho_{\mathrm{p}}$ in various solvents can be considered purely in terms of the aggregation behavior of PBLG. For example, the value of 0.94 for $\rho_{\mathrm{s}} / \rho_{\mathrm{p}}$ of

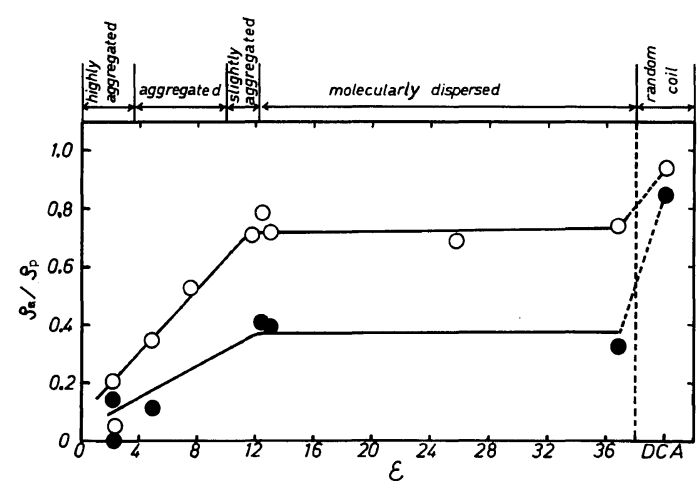

Figure 2. Dependence of $\rho_{\mathrm{s}} / \rho_{\mathrm{p}}$ for the apparent rotational relaxation time of the terminal group of PBLG on the dielectric constant of various solvents: $(O)$, PBLG260RhB; (O), PBLG480RhB.
PBLG260RhB in DCA, indicates that the mean rotational relaxation time of the rhodamine B group attached to PBLG chain end is nearly equal to that of RhB-NCS, showing the random coil behavior of PBLG. In contrast, the values of 0.21 for $\rho_{\mathrm{s}} / \rho_{\mathrm{p}}$ of PBLG260RhB in


Figure 3. Graph for the emission anisotropy against the composition of dioxane, benzene, chloroform, and benzyl alcohol-DCA and DMF, and DCA-DMF mixtures: (O), RhB-NCS; (๑), PBLG260RhB; ( $)$ ), PBLG480RhB. 
dioxane indicate that the mean rotational relaxation time of rhodamine B group attached to PBLG chain end is about five times that of RhB-NCS, showing the aggregation behavior of PBLG. In Table I, the relationships between the $\rho_{\mathrm{s}} / \rho_{\mathrm{p}}$ values and the behavior of PBLG in each solvent are in fair agreement with reported data ${ }^{18-20,24}$ except for that in benzyl alcohol. In our experiment, it may thus be presumed that the terminal rhodamine $B$ group as a chromophore is tightly bound to the PBLG chain end.

In Figure 2, the mean rotational relaxation times of PBLG480RhB are larger than that of PBLG260RhB in various helicogenic solvents because the mean rotational relaxation time of the longer rod-like polymer is larger than that of the shorter one. But in DCA (helix-breaking solvent) the $\rho_{\mathrm{s}} / \rho_{\mathrm{p}}$ values of PBLG480RhB roughly agree with that of PBLG280RhB because of the randam coil state of PBLG. Consequently, the fluorescence depolarization method for PBLG-RhB is very useful for studying aggregation and helix-coil transition behavior.

Only the $\rho_{\mathrm{s}} / \rho_{\mathrm{p}}$ value in benzyl alcohol (highly aggregation-solvent) in Table I does not agree with the reported data ${ }^{24}$ (PBLG is highly aggregated in benzyl alcohol.). Our data show that the extent of aggregation of PBLG is roughly proportional to the dielectric constant $\varepsilon$ up to $\varepsilon \approx 12$. If the aggregation of PBLG is the head-to-tail type in benzyl-alcohol, it can be presumed that the rhodamine $\mathrm{B}$ group attached to PBLG chain end disturbs the aggregation process of PBLG.

\section{Aggregation Behavior of $P B L G$ in the Series of Aggregation-Solvent-DMF Mixture}

Plots for the emission anisotropy $r$ of RhBNCS and PBLG-RhB against the composition of dioxane-DMF, benzene-DMF, and chloroform-DMF mixtures are shown in Figure 3, and the $\rho_{\mathrm{s}} / \rho_{\mathrm{p}}$ in the same solvent mixtures, and benzyl alcohol-DMF mixture are shown in Figures 4, 5, 6, and 7, re- spectively. PBLG is aggregated in dioxane, benzene, and chloroform and dispersed in DMF and thus, the $\rho_{\mathrm{s}} / \rho_{\mathrm{p}}$ values at various compositions of aggregation-solvent-DMF mixtures indicate the degree of aggregation of PBLG.

In the dioxane or benzene-DMF mixtures, the degree of PBLG aggregation gradually

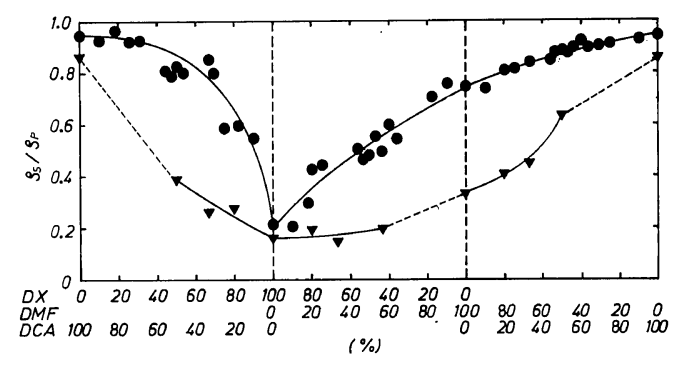

Figure 4. Dependence of $\rho_{\mathrm{s}} / \rho_{\mathrm{p}}$ for the rotational relaxation time of PBLG-RhB on the composition of dioxane-DMF, dioxane-DCA, and DMF-DCA mixtures: (○), PBLG260RhB; ( $\nabla)$, PBLG480RhB.

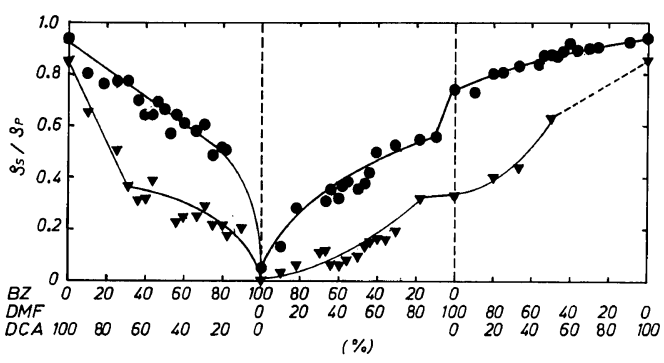

Figure 5. Dependence of $\rho_{\mathrm{s}} / \rho_{\mathrm{p}}$ for the rotational relaxation time of PBLG-RhB on the composition of benzene-DMF, benzene-DCA, and DMF-DCA mixtures: (○), PBLG260RhB; ( $)$ ), PBLG480RhB.



Figure 6. Dependence of $\rho_{\mathrm{s}} / \rho_{\mathrm{p}}$ for the rotational relaxation time of PBLG-RhB on the composition of chloroform-DMF, chloroform-DCA, and DMF-DCA mixtures: (○), PBLG260RhB; ( $)$ ), PBLG480RhB. 


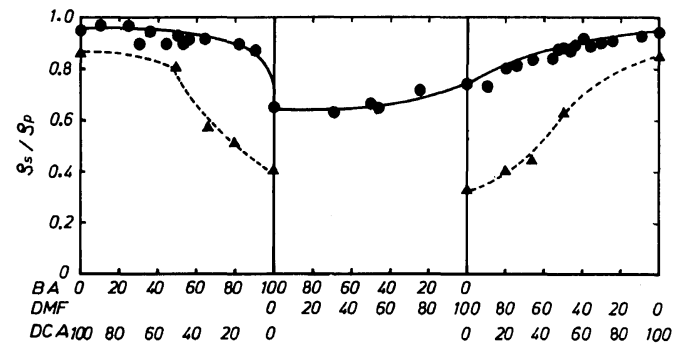

Figure 7. Dependence of $\rho_{\mathrm{s}} / \rho_{\mathrm{p}}$ for the rotational relaxation time of PBLG-RhB on the composition of benzyl alcohol-DMF, benzyl alcohol-DCA, and DMFDCA mixtures: (О), PBLG260RhB; ( $\nabla)$, PBLG480RhB.

decreases with increasing DMF percent, suggesting that the aggregation of PBLG cannot be destroyed immediately on addition of a small amount of DMF to the highly aggregation-solvents. The aggregation of PBLG is readily broken by the addition of a small amount of DMF to the chloroform solution in the chloroform-DMF mixture. These findings corresponds to the reported aggregation types ${ }^{24}$ (side-by-side type and head-to-tail type) of PBLG in dioxane, benzene, chloroform. In Figures 4 and 5, the curve of PBLG480RhB differs from that of PBLG260RhB in the dioxane and benzeneDMF mixtures, indicating that the aggregation of higher molecular-weight PBLG cannot be easily destroyed by the addition of DMF to the highly aggregation-solvents compared with that of a lower one.

\section{Helix-Coil Transition Behavior of PBLG in DMF-DCA Mixtures}

A similar figure of $\rho_{\mathrm{s}} / \rho_{\mathrm{p}}$ against the composition of DMF-DCA mixtures is attached to each of Figures 4-7. This enable us to compare the different composition of a mixed solvent in the same $\rho_{\mathrm{s}} / \rho_{\mathrm{p}}$. In the case of PBLG260RhB, the $\rho_{\mathrm{s}} / \rho_{\mathrm{p}}$ values gradually increase with increasing composition of DCA. In other words, the helix form in the vicinity of terminal group of PBLG is gradually transformed into a random-coil with increasing content of DCA. The curve of PBLG480RhB for $\rho_{\mathrm{s}} / \rho_{\mathrm{p}} v s$. the composition of DMF-DCA mixtures rises more steeply in $0-50 \%$ DCA region than in $50-100 \%$ DCA region. The somewhat sharp increase in $\rho_{\mathrm{s}} / \rho_{\mathrm{p}}$ value at 0 $50 \%$ DCA may be ascribed to the effect of dissociation of the aggregation of PBLG, suggesting it to be sightly aggregated even in DMF (molecularly dispersion-solvent ${ }^{24}$ ).

Aggregation and Helix-Coil Transition of $P B L G$ in the Series of AggregationSolvents-DCA Mixtures

The plots of $\rho_{\mathrm{s}} / \rho_{\mathrm{p}}$ against the composition of dioxane-DCA, benzene-DCA, chloroformDCA, and benzyl alcohol-DCA mixtures are also shown in Figures 4, 5, 6, and 7, respectively. The DCA is well-known as a helixbreaking solvent for PBLG. As evident from Figures $4-7$, the $\rho_{\mathrm{s}} / \rho_{\mathrm{p}}$ value of PBLG-RhB increases with increasing DCA percent. Moreover, the slope with DCA is sharper than that with DMF at an early stage. The difference in the slopes of DCA and DMF can be explained not only by the fact that DCA is a helix-breaking solvent for PBLG but also that the aggregation-breaking power of DCA for PBLG is larger than that of DMF.

In Figure 4 , the $\rho_{\mathrm{s}} / \rho_{\mathrm{p}}$ value for PBLG$260 \mathrm{RhB}$ in the dioxane-DCA mixture increases sharply at $0-30 \%$ DCA. The $\rho_{\mathrm{s}} / \rho_{\mathrm{p}}$ value for the molecularly dispersed PBLG260RhB must agree with that in DMF, that is, $\rho_{\mathrm{s}} / \rho_{\mathrm{p}}=$ 0.74 (Table I). Thus, the change in $\rho_{\mathrm{s}} / \rho_{\mathrm{p}}$ at an early stage can be attributed to the aggregation-breaking process of PBLG by DCA. In contrast, in case of PBLG480RhB, $\rho_{\mathrm{s}} / \rho_{\mathrm{p}}$ gradually increases with increasing composition of DCA up to $\rho_{\mathrm{s}} / \rho_{\mathrm{p}}=0.33$ (value of PBLG480RhB in DMF). This change suggests the existence of a stronger aggregation for PBLG480RhB compared with that of PBLG260$\mathrm{RhB}$ in dioxane.

In Figure 5, $\rho_{\mathrm{s}} / \rho_{\mathrm{p}}$ increases rather sharply in two regions, i.e., at $0-20 \%$ and $80-100 \%$ DCA for PBLG260RhB and $0-10 \%$ and $60-$ $100 \%$ DCA for PBLG480RhB. As noted 
above, the aggregation-breaking process of PBLG continues up to $\rho_{\mathrm{s}} / \rho_{\mathrm{p}}=0.74$ (PBLG260RhB) or 0.33 (PBLG480RhB). Consequently, the result in Figure 5 shows that the aggregation of PBLG cannot be broken up to about $70 \%$ DCA for PBLG260RhB and PBLG480RhB. The somewhat sharp increase in the $\rho_{\mathrm{s}} / \rho_{\mathrm{p}}$ value at $70-100 \%$ DCA may be ascribed to the helix-coil transition behavior of PBLG.

In Figure 6 , the $\rho_{\mathrm{s}} / \rho_{\mathrm{p}}$ value in chloroformDCA mixture gradually increases up to $\rho_{\mathrm{s}} /$ $\rho_{\mathrm{p}}=0.74$ (PBLG260RhB) or 0.33 (PBLG$480 \mathrm{RhB})$ in the region of $0-40 \% \mathrm{DCA}$, and sharply increases in the region of 60 $80 \%$ DCA again. The former behavior is the aggregation-breaking process, and the later behavior can be ascribed to the helix-coli transition process, since it corresponds to the helix-coil transition region of PBLG in chloroform-DCA mixture observed by several other methods such as ORD, ${ }^{25}$ intrinsic viscosity, ${ }^{25}$ laser light scattering. ${ }^{26}$

In Figure 7, the $\rho_{\mathrm{s}} / \rho_{\mathrm{p}}$ value sharply increases at $0-10 \%$ DCA for PBLG $260 \mathrm{RhB}$ or $0-50 \%$ DCA for PBLG480RhB. Moreover, this sharp increase in the $\rho_{\mathrm{s}} / \rho_{\mathrm{p}}$ value is above both 0.74 (PBLG260RhB) and 0.33 (PBLG480RhB). These changes may primarily indicate the helix-coil transition behavior of PBLG. Our experiment suggests that the aggregation of PBLG is either absent or undetectable by this fluorescence depolarization method in benzyl alcohol solution, as opposed to the highly aggregation-solvent reported by many authors. ${ }^{11,25}$

\section{Summary of Aggregation and Helix-coil} Transition Behavior of PBLG in the Various Mixed Solvents

(1) In high aggregation-solvents (dioxane and benzene), the aggregation of PBLG cannot be destroyed immediately on addition of a small amount of DMF. Moreover, though the increase in $\rho_{\mathrm{s}} / \rho_{\mathrm{p}}$ is larger by the addition of DCA than that of DMF, the aggregation of
PBLG cannot be destroyed completely up to $40 \%$ DCA (in dioxane) and 70\% (in benzene).

(2) The aggregation of PBLG is readily broken down by the addition of DMF and DCA to the chloform solution. The helix-coil transition behavior of PBLG appears at the region of $60-80 \%$ DCA in a chloroformDCA mixture.

(3) In the benzyl alcohol-DCA mixture, the aggregation behavior of PBLG cannot be detected by our experimental method.

(4) In the DMF-DCA mixture, the apparent helix-coil transition behavior of PBLG proceeds gradually. This behavior may be attributable to the helix-coil transition only in the vicinity of a chain end group of PBLG. In contrast, the apparent helix-coil transition behavior of PBLG proceeds sharply in the benzene-DCA and chloroform-DCA mixtures. This difference in helix-coil transition behavior in DMF-DCA and benzene-DCA mixtures is discussed briefly in next section.

(5) In the dioxane, benzene, and chloroform-DCA mixtures, the curve representing the $\rho_{\mathrm{s}} / \rho_{\mathrm{p}} v s$. DCA content relationship is temporarily flat in a certain region of DCA content. The $\rho_{\mathrm{s}} / \rho_{\mathrm{p}}$ value in this region roughly agrees with that in the DMF solution. Thus, the initial upward curve leading to the flat curve may possibly be due to the dissociation process of aggregated PBLG.

\section{Analysis for Aggregation and Helix-Coil Transition Behavior of PBLG in Various Mixed Solvents}

To facilitate an understanding of the relation between the aggregation and helix-coil transition behavior of PBLG, the apparent aggregation-breaking (dissociation) $A_{x}$ and helix-breaking $H_{x}$ ratios at any specified composition of various mixed solvents were calculated by,

$$
\begin{aligned}
& A_{x}=\left(R_{x}-R_{\mathrm{AS}}\right) /\left(R_{\mathrm{DMF}}-R_{\mathrm{AS}}\right) \times 100(\%) \\
& H_{x}=\left(R_{x}-R_{\mathrm{DMF}}\right) /\left(R_{\mathrm{DCA}}-R_{\mathrm{DMF}}\right) \times 100(\%)
\end{aligned}
$$




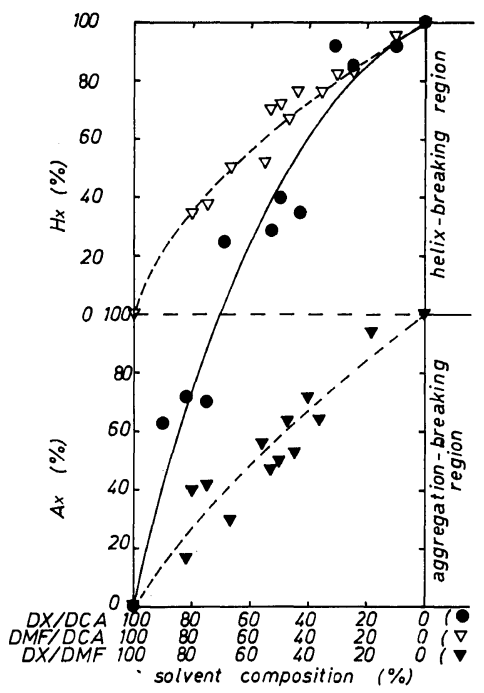

Figure 8. Graph for the aggregation-breaking ratio $A_{x}$ and helix-breaking (dissociation) ratio in the vicinity of the chain end group attached to PBLG260 $H_{x}$ against the composition of dioxane-DMF, dioxane-DCA, and DMF-DCA mixtures: $(\boldsymbol{\nabla})$, composition of the dioxaneDMF mixture; ( $)$, composition of the dioxane-DCA mixture; $(\nabla)$, composition of the DMF-DCA mixture.

where $R_{\mathrm{AS}}, R_{\mathrm{DMF}}$, and $R_{\mathrm{DCA}}$ are the $\rho_{\mathrm{s}} / \rho_{\mathrm{p}}$ values in the aggregation-solvents (dioxane, benzene, and chloroform), DMF, and DCA, respectively, and the subscript $x$ is used as necessary to denote a certain composition of dioxane$\mathrm{DMF}$ or DCA, benzene-DMF or DCA, chloroform-DMF or DCA, and DMF-DCA mixtures.

The plots of $A_{x}, H_{x}$, and $A_{x}$ plus $H_{x}$ for PBLG260RhB against the composition of various mixed solvents are shown in Figures 810. According to each curve for $A_{x} v s$. the composition of the aggregation-solvent-DMF in Figures 8-10, the degree of the aggregation-breaking ratio $A_{x}$ in chloroformDMF mixture is larger than that in dioxaneDMF and benzene-DMF mixtures. In the DMF-DCA mixture of each figure, $H_{x}$ gradually increases with increasing DCA percent.

The helix-coil transition behavior in the vicinity of the terminal group of PBLG is apparent from each curve form in the aggregation-solvent-DCA mixtures in Figures

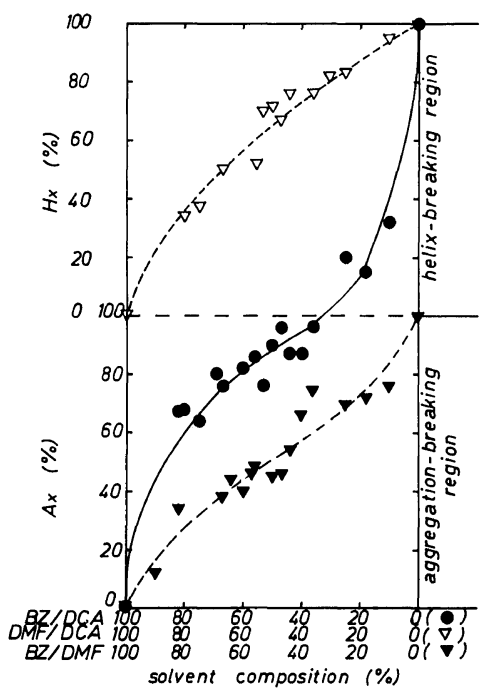

Figure 9. Graph for the aggregation-breaking ratio $A_{x}$ and helix-breaking (dissociation) ratio in the vicinity of the chain end group attached to PBLG260 $H_{x}$ against the composition of benzene-DMF, benzene-DCA, and DMF-DCA mixtures: $(\boldsymbol{\nabla})$, composition of the benzeneDMF mixture; (O), composition of the benzene-DCA mixture; $(\nabla)$, composition of the DMF-DCA mixture.

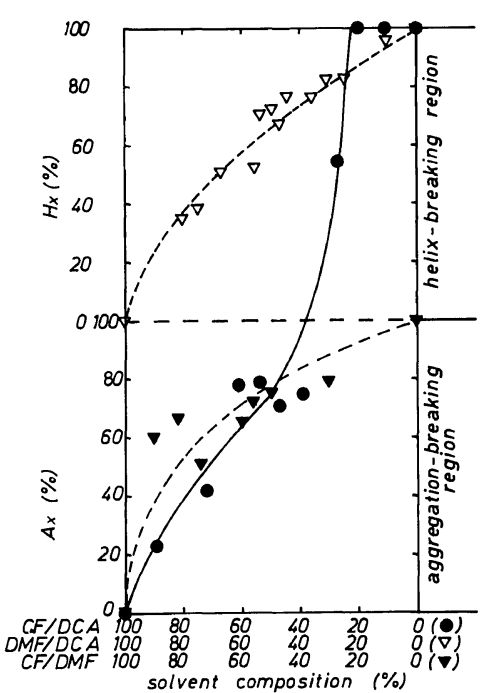

Figure 10. Graph for the aggregation-breaking ratio $A_{x}$ and helix-breaking (dissociation) ratio in the vicinity of the chain end group attached to PBLG260 $H_{x}$ against the composition of chloroform-DMF, chloroformDCA, and DMF-DCA mixtures: $(\boldsymbol{\nabla})$, composition of the chloroform-DMF mixture; (O), composition of the chloroform-DCA mixture; $(\nabla)$, composition of the DMF-DCA mixture. 
8-10. In Figure 8 (dioxane-DCA mixture), the curve for the dioxane-DCA mixture shows that helix-coil transition behavior in the vicinity of the terminal group of PBLG260$\mathrm{RhB}$ occurs at about $40 \%$ DCA. But such behavior in the benzene-DCA and chloroformDCA mixtures (in Figures 9 and 10) cannot occur up to about $70 \%$ DCA (benzene) and $60 \%$ DCA (chloroform). These finding suggest that helix-coil transition behavior in the vicinity of the chain end group of PBLG is inhibited by the aggregation behavior of PBLG. That is, the helix-coil transition can occur immediately after PBLG has been completely dissociated from DCA.

In conclusion, the measurement of the emission anisotropy $r$ of rhodamine B chemically bound to the terminal amino group of PBLG in various mixed solvents shows $H_{x}$ in the vicinity of the chain end group of PBLG to gradually increases in DMF-DCA mixture with increasing DCA percent, and $A_{x}$ to gradually increase in highly aggregation-solvents (dioxane and benzene) with increasing DMF percent. Helix-coil transitions in the vicinity of the chain end goup of PBLG cannot occur up to about $60-70 \%$ DCA in the benzene-DCA and chloroform-DCA mixtures. Helix-coil transition curves in the vicinity of the terminal group of PBLG increase almost exclusively from $H_{x}=0 \%$ to $100 \%$ in $80-100 \%$ DCA (benzene) and $60-80 \%$ DCA (chloroform). Thus, helixcoil transition behavior in the vicinity of the chain end group of PBLG may occur easily following the complete break down of PBLG aggregation by the addition of DCA. It is likely that helix-coil transition behavior in the aggregation-solvents-DCA mixtures is inhibited by the aggregation of PBLG.

\section{REFERENCES}

1. H. Ushiki and I. Mita, Polym. J., 13, 837 (1981).

2. E. L. Wee and W. G. Miller, J. Phys. Chem., 77, 182 (1973).

3. A. Tsutsumi, S. Kon, and K. Hikichi, Polym. Prepr. Jpn., 30, 255 (1981).

4. F. Perrin, Ann. Phys., 12, 169 (1929).

5. K. Horie, I. Mita, J. Kawabata, S. Nakahama, A. Hirao, and N. Yamazaki, Polym. J., 12, 319 (1980).

6. Y. Nishijima, J. Polym. Sci., C, 31, 353 (1970).

7. L. Monnerie and J. P. Jarry, Ann. New York Acad. Sci., 366, 328 (1981).

8. N. Kasparyan-tardiveau, B. Valeur, L. Monnerie, and I. Mita, Polymer, 24, 205 (1983).

9. A. Teramoto and H. Fujita, Adv. Polym. Sci., 18, 65 (1975).

10. A. Teramoto and H. Fujita, J. Macromol. Sci., Rev., Macromol. Chem., C, 15, 165 (1976).

11. N. Sayama, K. Kida, T. Norisuye, A. Teramoto, and H. Fujita, Polym. J., 3, 538 (1971).

12. A. Teramoto, K. Nakagawa, and H. Fujita, J. Chem. Phys., 46, 4197 (1967).

13. J. N. C. Ford and W. Lee, J. Chem. Phys., 50, 3098 (1969).

14. G. Schwarz and J. Seeling, Biopolymers, 6, 1263 (1978).

15. M. Hatano, T. Enomoto, I. Ito, and M. Yoneyama, Bull. Chem. Soc., Jpn., 46, 3698 (1973).

16. M. Morcellet and C. Loucheux, Eur. Polym. J., 14, 697 (1978).

17. A. Wada, J. Polym. Sci., 45, 145 (1960).

18. K. Watanabe, Nippon Kagaku Zasshi, 86, 65 (1965).

19. H. Kihara, Polym. J., 7, 406 (1975).

20. H. Kihara, Polym. J., 9, 443 (1977).

21. von J. Gerber and H. G. Elias, Makromol. Chem., 112, 142 (1968).

22. T. Iio, Y. Iwashita, and H. Watanabe, Bull. Chem. Soc., Jpn., 45, 2206 (1972).

23. P. Doty, J. Am. Chem. Soc., 78, 947 (1956).

24. R. Sakamoto and M. Watanabe, Polym. Prepr., Jpn., 28, 1430 (1981).

25. P. Doty, Collect. Czech. Chem. Commun., 25, 511 (1957).

26. J. N. C. Ford, W. Lee, and F. E. Karasz, J. Chem. Phys., 50, 3098 (1969). 